\title{
Nutlin-3 reverses the epithelial-mesenchymal transition in gemcitabine-resistant hepatocellular carcinoma cells
}

\author{
QIONG WU ${ }^{1 *}$, XI WANG ${ }^{2 *}$, JING LIU $^{1}$, JIYUE ZHENG ${ }^{1}$, YANG LIU $^{1}$, \\ YUMEI LI ${ }^{1}$, FANG SU ${ }^{1}$, WEILI OU ${ }^{1}$ and RUI WANG ${ }^{1}$ \\ ${ }^{1}$ Department of Medical Oncology, The First Affiliated Hospital of Bengbu Medical College, Bengbu, Anhui 233004; \\ ${ }^{2}$ Department of Oncology, The 117th Hospital of PLA, Hangzhou, Zhejiang 310013, P.R. China
}

Received January 29, 2016; Accepted March 5, 2016

DOI: $10.3892 /$ or.2016.4920

\begin{abstract}
Nutlin-3, a small molecule regulator of the tumor suppressor p53, targets the interaction between p53 and murine double minute 2 (MDM2) thereby promoting stabilization of p53 and subsequent p53-dependent induction of apoptosis and cell cycle arrest. Recent studies have demonstrated that Nutlin-3 plays a critical role in regulating tumor cell migration, invasion, metastasis, and drug resistance. Although these studies identified various biological functions of Nutlin-3, our understanding of the exact molecular mechanisms of Nutlin-3-mediated antitumor activity remains incomplete. In this study, we elucidated a role of Nutlin-3 in reversing the epithelial-mesenchymal transition (EMT) in gemcitabine-resistant (GR) hepatocellular carcinoma (HCC) cells. We assessed the effect of Nutlin-3 treatment on cell growth, migration, and invasion in both parental HCC cells and GR HCC cells. Moreover, we detected the expression of EMT markers in GR HCC cells treated with Nutlin-3 by real-time RT-PCR and western blot analysis, respectively. We found that Nutlin-3 inhibited cell migration and invasion in the GR HCC cells. Additionally, Nutlin-3 treatment increased E-cadherin protein levels, but decreased the protein levels of vimentin, Snail and Slug in the GR HCC cells. Furthermore, we found that Smad2 was highly expressed in the GR HCC cells compared with their parental HCC cells, and Nutlin-3 treatment downregulated Smad2 expression in the GR HCC cells. Depletion of Smad2 retarded cell migration and regulated the expression of EMT markers in GR HCC cells similarly to Nutlin-3 treatment. Our findings highlight an important role of Nutlin-3 in reversing EMT in GR cells through regulation of
\end{abstract}

Correspondence to: Dr Rui Wang, Department of Medical Oncology, The First Affiliated Hospital of Bengbu Medical College, 287 Changhuai Road, Bengbu, Anhui 233004, P.R. China

E-mail: ruiwang84@sina.com

*Contributed equally

Key words: hepatocellular carcinoma, gemcitabine, EMT, Nutlin-3, MDM2
Smad 2 expression, suggesting that Nutlin-3 could be a potential agent for the treatment of HCC patients with gemcitabine resistance.

\section{Introduction}

Hepatocellular carcinoma (HCC) is the fifth most common form of cancer worldwide (1). It is estimated that more than 35,600 new cases of HCC were reported and 24,550 people died due to HCC in 2015 in the USA (2). Since most HCC patients are diagnosed at a late stage, surgical resection and liver transplantation fail to significantly increase the 5-year survival rate (3). Chemotherapeutic drugs are useful for advanced stage HCC patients, and clinical trials have revealed that advanced HCC patients treated with sorafenib have extended median survival by 3 months $(4,5)$. One recent study demonstrated that fluorouracil, leucovorin, and oxaliplatin (FOLFOX4) treatment conferred some benefit to patients with advanced HCC (6). Recently, one study validated gemcitabine and oxaliplatin (GEMOX) as effective with manageable toxicity in advanced HCC patients (7). However, due to the fact that many patients develop drug resistance to standard chemotherapy, these treatments often do not improve patient outcomes $(8,9)$. Therefore, it is important to develop new therapeutic agents to increase drug sensitivity to chemotherapy and achieve better treatment outcomes for HCC patients.

Emerging evidence has demonstrated that epithelial-mesenchymal transition (EMT) is critically involved in chemoresistance in human cancers including HCC (10). It has been well documented that during the EMT process, epithelial cells convert into mesenchymal cells due to loss of epithelial cell-cell junction (11). Mechanistically, expression of epithelial markers including E-cadherin and $\gamma$-catenin is downregulated, whereas the mesenchymal markers such as vimentin, Twist, Slug, and Snail are upregulated. EMT-type cells acquire enhanced migration and invasion leading to more enhanced metastasis (12). In addition, there appears to be a direct connection between EMT and the development of drug resistance (13). For example, multiple studies have revealed that gemcitabine-resistant (GR) cells acquired EMT characteristics in pancreatic cancer and HCC cells (14-17). Specifically, several signaling pathways have been identified to be involved in GR-mediated EMT including Notch-1, 
platelet-derived growth factor-D (PDGF-D), and hypoxia inducible factor-1 $\alpha$ (HIF-1 $\alpha$ ) pathways (14-17). Thus, targeting these pathways could reverse EMT and increase gemcitabine sensitivity in human cancers. Therefore, in-depth investigation of the molecular mechanisms governing GR-induced EMT would aid the discovery of novel strategies for the treatment of HCC patients.

Previous studies have implicated the p53 pathway as an essential regulator of EMT and drug resistance (18-20). For instance, $\mathrm{p} 53$ inactivation in breast cancer cells promoted EMT and increased the susceptibility to 3-bromopyruvate (18). Lin et al also identified that p53 modulated NF-kB-mediated EMT in head and neck squamous cell carcinoma (19). Another study identified that targeting ephrin-B2 might enhance the therapeutic potential of DNA-damaging chemotherapeutic agents in human tumors harboring mutant p53 (20). It is well established that $\mathrm{p} 53$ is negatively regulated by the murine double minute 2 (MDM2) oncoprotein (21). Thus, it was logical to develop small molecules that selectively disrupt the interaction between MDM2 and p53, leading to a restoration of $\mathrm{p} 53$ function in tumor cells with wild-type p53 (22). To this end, Nutlin-3 was identified as a selective inhibitor of the p53-MDM2 interaction and exhibited antitumor activities in a variety of human malignances (23). By activating the p53 pathway, Nutlin-3 enhances cellular apoptosis and inhibits cell growth (24). Recent studies indicate that Nutlin-3 also exerts its anticancer functions partly through negative regulation of migration, invasion and drug resistance (25-27), however further studies are required to fully understand the molecular mechanisms driving Nutlin-3-induced inhibition of tumorigenesis.

Here we report that Nutlin-3 inhibited cell migration and invasion in GR HCC cells, which acquired EMT features. Moreover, to further define the mechanisms behind the tumor-suppressive functions of Nutlin-3, we identified that treatment with Nutlin-3 led to upregulation of E-cadherin and downregulation of mesenchymal markers including vimentin, Snail and Slug in GR HCC cells. Mechanistically, our results revealed that Smad2 was upregulated in GR HCC cells compared with their parental cells, and that Nutlin-3 inhibited the expression of Smad2 in GR HCC cells. Consistent with this notion, depletion of Smad2 suppressed cell migration and regulated the expression of EMT markers in GR HCC cells. These results indicate that Nutlin-3 could reverse EMT in GR HCC cells, providing the rational for the use of Nutlin-3 as a potential treatment for HCC patients.

\section{Materials and methods}

Cell culture, reagents and antibodies. Human HepG2 and SMMC-7721 cell lines were cultured at $37^{\circ} \mathrm{C}$ in $5 \% \mathrm{CO}_{2}$ in Dulbecco's modified Eagle's medium (DMEM; Gibco, Gaithersburg, MD, USA) supplemented with $10 \%$ fetal bovine serum. HepG2 GR and SMMC-7721 GR cell lines were established in our laboratory as described previously (14). Gemcitabine, [3-(4,5-dimethylthiazol-2-yl)-2,5-diphenyltetrazolium bromide] (MTT), and Nutlin-3 were obtained from Sigma (St. Louis, MO, USA). Antibodies against vimentin and E-cadherin were obtained from Abcam. Antibodies against Snail, Slug, Smad2, and GAPDH were obtained from Santa Cruz Biotechnology (Santa Cruz, CA, USA).
MTT assay. Cells were seeded at equal densities into 96-well culture plates and incubated overnight. Cells were then treated with the indicated concentrations of Nutlin-3 for $48 \mathrm{~h}$. MTT assay was conducted as previously described (28). Cell growth inhibition curves were generated using the cell survival rates. The concentrations of Nutlin-3 including IC 30, IC50, and IC70 were calculated and used in the subsequent experiments.

Wound healing assay. HepG2 GR and SMMC-7721 GR cells were cultured in 6-well plates and grown to confluency. After cells converged almost $100 \%$, monolayers of cells were scratched with yellow pipette tips. Then the cells were washed with PBS and medium was added with different concentrations of Nutlin-3. The scratched area was photographed with a microscope at 0 and $20 \mathrm{~h}$, respectively, to observe the cell motility ability.

Transwell migration and invasion assays. The migration assay was carried out in chambers without matrix gel and the invasion assay was performed in a chamber with $50 \mu 1$ matrix gel. Briefly, the HepG2 GR and SMMC-7721 GR cells were seeded in the upper chamber with $200 \mu 1$ serum-free medium and the indicated concentrations of Nutlin-3 and $500 \mu \mathrm{l}$ complete medium in the lower chamber with the same concentration of Nutlin-3. After incubation for $24 \mathrm{~h}$, the membrane of the chamber was immobilized with methanol and strained with $1 \%$ crystal violet. Cells were counted and photographed with a microscope.

Cell attachment and detachment assays. For the attachment assay, HepG2 GR and SMMC-7721 GR cells, treated with the indicated concentrations of Nutlin-3, were seeded in 24-well plates at $5 \times 10^{4}$ cells per well. Unattached cells were removed after a 1-h incubation, and the attached cells were counted after $0.25 \%$ trypsinization. The data are presented as a percentage of the attached cells compared to total cells. For the cell detachment assay, after a 24-h incubation, the cells were incubated with $0.05 \%$ trypsin for 3 min to detach the cells. Culture medium was then added to inactivate the trypsin and the detached cells were collected. The remaining cells were incubated with $0.25 \%$ trypsin to detach and counted. The data are presented as a percentage of the detached cells to total cells.

Real-time RT-PCR. Total RNA from the HepG2 GR and SMMC-7721 GR cells treated with Nutlin-3 was isolated with TRIzol or with the RNeasy Mini kit and RNase-free DNase set according to the manufacturer's protocols. RNA was reversed-transcribed into cDNA by RevertAid First Strand cDNA Synthesis kit (Thermofisher Scientific, Inc.) according to the manufacturer's protocol. The primers used in the PCR reactions are listed in Table I. The expression of GAPDH was used as internal control. RT-PCR amplifications were performed as previously described (28).

Western blot analysis. Harvested cells were washed by PBS and lysed with protein lysis buffer, and protein concentrations were measured by Bradford assay reagent. Equal amounts of protein were separated by sodium dodecyl sulfate (SDS)-polyacrylamide gel electrophoresis (SDS-PAGE) 

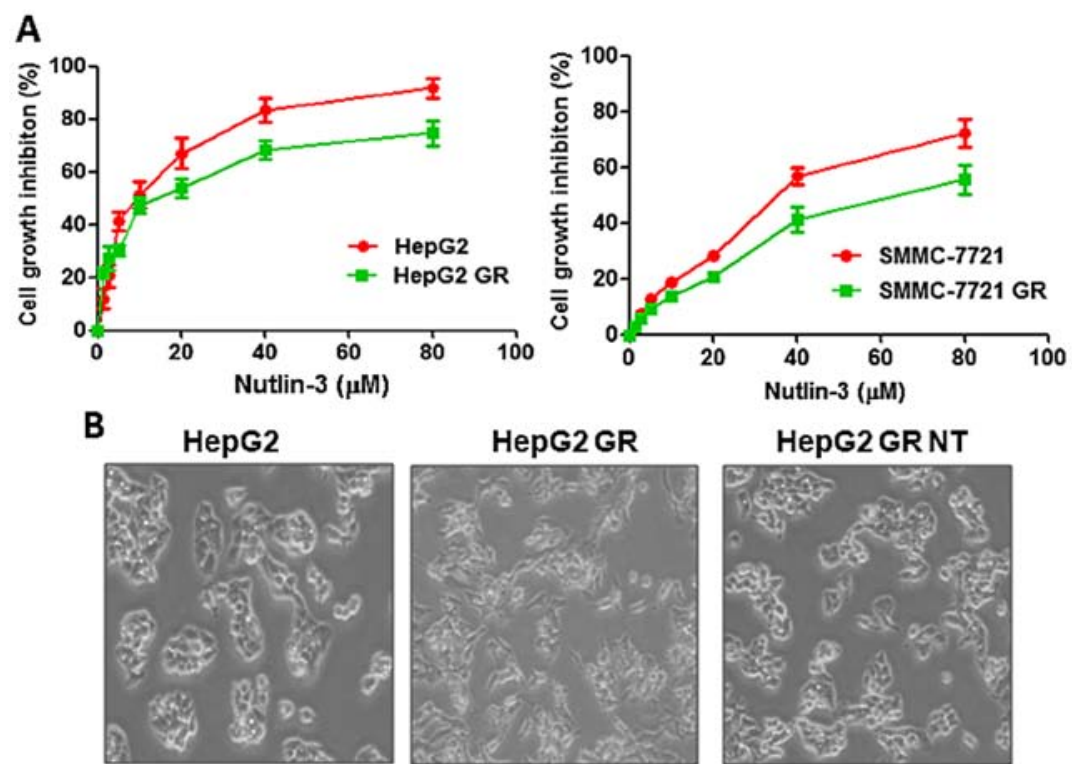

HepG2 GR NT

SMMC

SMMC GR
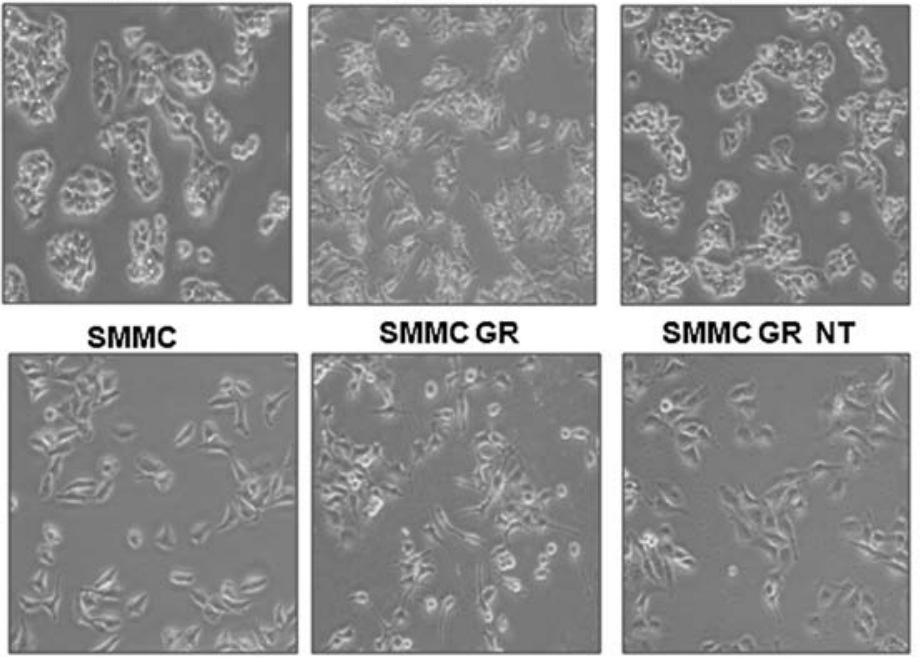

SMMC GR NT

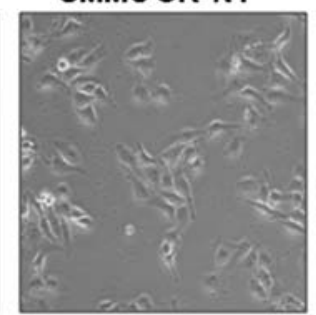

Figure 1. Nutlin-3 inhibits cell proliferation and alters the morphology of GR HCC cells. (A) MTT assay was performed in parental HCC cells (HepG2 and SMMC-7721) and gemcitabine-resistant HCC (GR HepG2 and GR SMMC-7721) cells after treatment with the indicated concentrations of Nutlin-3 for $48 \mathrm{~h}$. (B) Cell morphology as observed by microscopy in the HCC and GR HCC cells treated with IC70 Nutlin-3 (NT) for $48 \mathrm{~h}$.

Table I. Primer sequences used for RT-PCR.

\begin{tabular}{|c|c|}
\hline Name & Sequence \\
\hline E-cadherin & $\begin{array}{l}\text { Sense: 5'-GAAGTGTCCGAGGACTTTGG-3' } \\
\text { Antisense: 5'-CAGTGTCTCTCCAAATCCGATA-3' }\end{array}$ \\
\hline Vimentin & $\begin{array}{l}\text { Sense: 5'-TGTCCAAATCGATGTGGATGTTTC-3' } \\
\text { Antisense: 5'-TTGTACCATTCTTCTGCCTCCTG-3' }\end{array}$ \\
\hline Slug & $\begin{array}{l}\text { Sense: 5'-CATGCCTGTCATACCACAAC-3' } \\
\text { Antisense: 5'-GGTGTCAGATGGAGGAGGG-3' }\end{array}$ \\
\hline Snail & $\begin{array}{l}\text { Sense: 5'-CGGAAGCCTAACTACAGCGA-3' } \\
\text { Antisense: 5'-GGACAGAGTCCCAGATGAGC-3' }\end{array}$ \\
\hline GAPDH & $\begin{array}{l}\text { Sense: 5'-CAGCCTCAAGATCATCAGCA-3' } \\
\text { Antisense: 5'-TGTGGTCATGAGTCCTTCCA-3' }\end{array}$ \\
\hline
\end{tabular}

and then transferred onto polyvinylidene fluoride (PVDF) membranes. The membranes where incubated with the primary antibody at $4^{\circ} \mathrm{C}$ overnight. After washing with TBS-Tween-20, the membranes were incubated with the secondary antibody conjugated to HRP at room temperature for $1 \mathrm{~h}$. The membranes were washed again in TBS-Tween-20, and protein was detected by electrochemiluminescence (ECL) assay.

Transfection. Cells were seeded in 6-well plates and transfected with control siRNA or Smad2 siRNAs (GenePharma,
Shanghai, China) using Lipofectamine 2000 as previously described (28).

Statistical analysis. All statistical analyses were conducted using SPSS17.0. Student's t-test was performed to evaluate statistical significance. Results are presented as mean \pm SD. $\mathrm{P}<0.05$ was considered as statistically significant.

\section{Results}

Nutlin-3 inhibits cell proliferation and alters the morphology of GR HCC cells. To determine whether Nutlin-3 inhibits cell proliferation in HCC cells, we performed MTT assays following Nutlin-3 treatment. We found that Nutlin-3 significantly inhibited cell proliferation in both the HepG2 and SMMC-7721 cells (Fig. 1A). Moreover, we observed that Nutlin-3 also suppressed cell proliferation in both the GR HepG2 and GR SMMC-7721 cells (Fig. 1A). Our results indicate that HCC cells are more sensitive to Nutlin-3 treatment compared with GR HCC cells (Fig. 1A). Specifically, IC30, IC50 and IC70 of Nutlin-3 in the GR HepG2 cells were 5, 18 and $70 \mu \mathrm{M}$, respectively. Whereas the IC70 of Nutlin-3 was $25 \mu \mathrm{M}$ in the HepG2 cells, suggesting that HepG2 cells were more sensitive to Nutlin-3 treatment (Fig. 1A, left panel). Similar results were observed in the SMMC-7721 cells (Fig. 1A, right panel). IC30, IC50 and IC70 of Nutlin-3 in the GR SMMC-7721 cells were 40, 59 and $80 \mu \mathrm{M}$, respectively. In subsequent experiments, we used IC30 and IC70 to explore the function of Nutlin-3 in the GR 
A
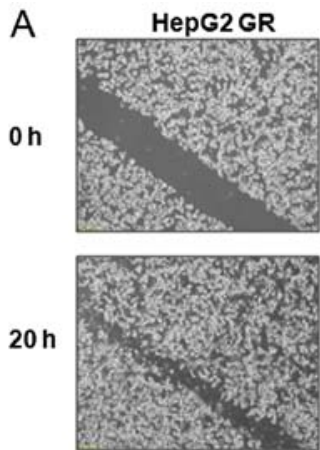

HepG2 GR

B

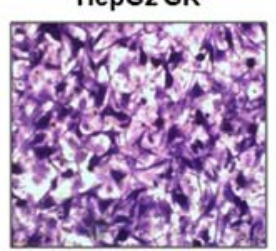

HepG2 GR

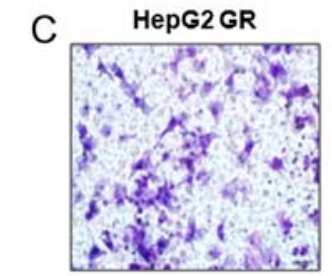

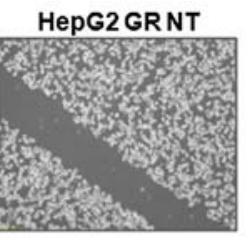

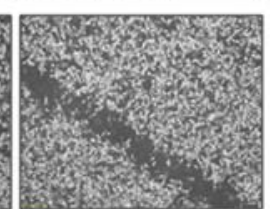

HepG2 GR NT

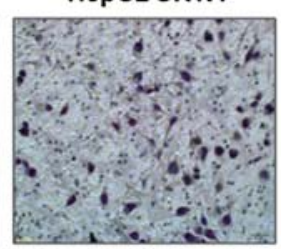

HepG2 GR NT

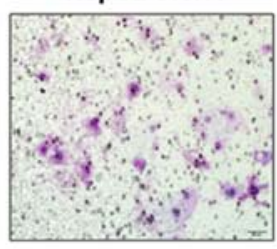

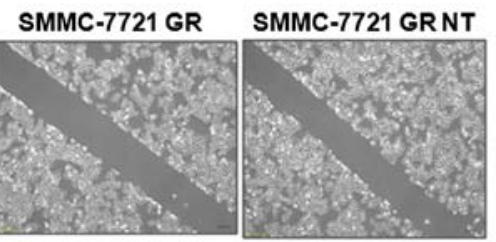

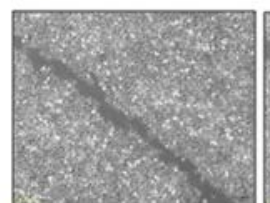

SMMC GR

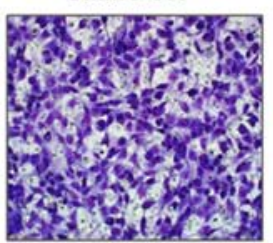

SMMC GR
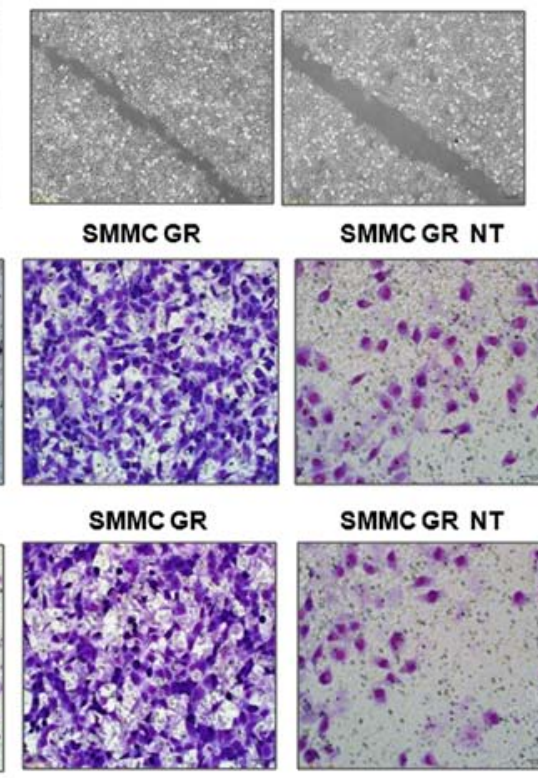

SMMC GR NT

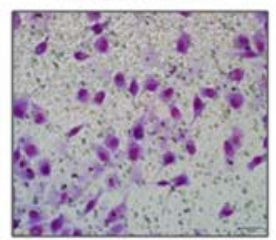

SMMC GR NT
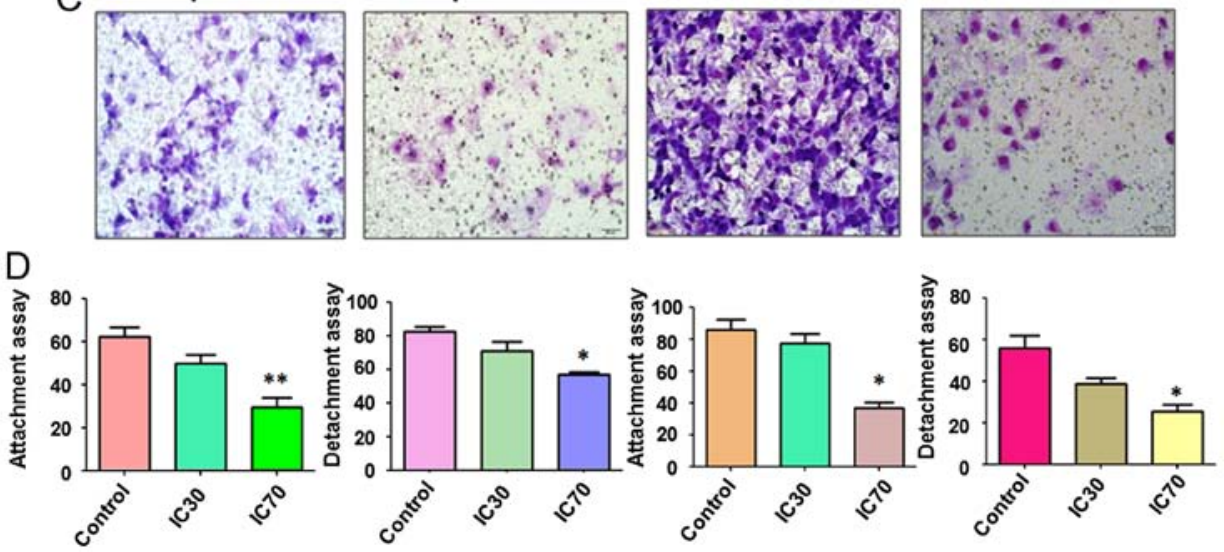

Figure 2. Nutlin-3 suppresses the motile activity of the GR HCC cells. (A) Wound healing assays to compare the migratory potential of GR HepG2 and GR SMMC-7721 cells that were treated with IC70 of Nutlin-3. NT, Nutlin-3. (B) Migration assay to measure the migratory capacity in GR HCC cells treated with IC70 of Nutlin-3 for $24 \mathrm{~h}$. (C) Invasion assay in GR HCC cells treated with IC70 Nutlin-3 for 24 h. (D) Cell attachment and detachment assays in GR HCC cells treated with IC70 Nutlin-3. ${ }^{*} \mathrm{P}<0.05,{ }^{* *} \mathrm{P}<0.01$ vs. control.

HCC cells. Our previous study demonstrated that GR HCC cells acquired an EMT phenotype (14). In line with this report, we observed the morphological change of GR HCC cells, which is indicative of EMT, such as increased pseudopodia, and longer, narrower and a more disperse morphology (Fig. 1B). Moreover, we found that Nutlin-3 treatment reversed this EMT phenotype (Fig. 1B). Specifically, in GR HCC cells, pseudopodia were reduced and cells became ovoid and clustered during growth following Nutlin-3 treatment (Fig. 1B). These results indicate that Nutlin-3 may regulate GR-induced EMT in HCC cells.

Nutlin-3 suppresses motility in GR HCC cells. To explore whether Nutlin-3 suppresses the motility of GR HCC cells, we performed wound healing assays to investigate the migration of GR HepG2 and GR SMMC-7721 cells following Nutlin-3 treatment. Our results from the wound healing assays demonstrated that Nutlin-3 markedly decreased cell migration in both the GR HepG2 and GR SMMC-7721 cells (Fig. 2A). To further validate this finding, migration and invasion assays were conducted to measure the cell migratory and invasive activity of the GR HCC cells treated with Nultin-3. We observed that Nutlin-3 significantly inhibited the migration (Fig. 2B) and invasion (Fig. 2C) in the GR HCC cells. Cell detachment and attachment are associated with cell motility, therefore we measured the cell attachment and detachment in the GR HCC cells following Nutlin-3 treatment. As demonstrated in Fig. 2D, Nutlin-3 treatment suppressed both cell attachment and detachment in the GR HepG2 and GR SMMC-7721 cells. Altogether, Nutlin-3 treatment may inhibit motility in GR HCC cells.

Nutlin-3 reverses EMT to MET in GR HCC cells. To further identify whether Nutlin-3 regulates specific EMT molecules in GR HCC cells, we measured the expression of markers of epithelial and mesenchymal phenotypes using RT-PCR and western blot analysis, respectively. Our RT-PCR results indicated that Nutlin-3 treatment increased E-cadherin mRNA levels, but decreased several mesenchymal markers at the mRNA level including vimentin, Snail and Slug in both GR HepG2 (Fig. 3A) and GR SMMC-7721 cells (Fig. 3B). Importantly, our western blot analysis confirmed that the protein level of E-cadherin was increased, whereas the expressions of vimentin, Snail and Slug were downregulated in the GR HCC cells following Nutlin-3 treatment (Fig. 3C). These observations indicated that Nutlin-3 reversed EMT to MET 
A

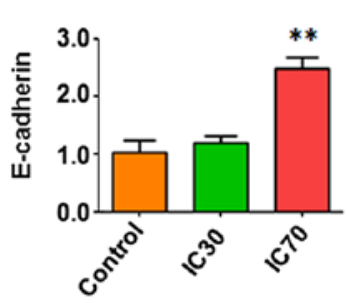

B

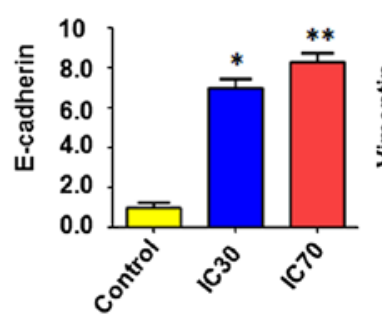

C
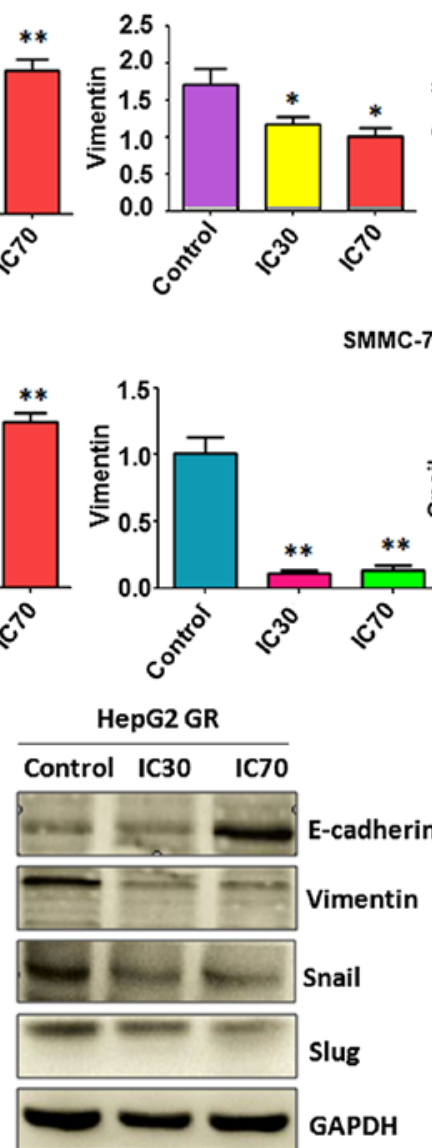

HepG2 GR
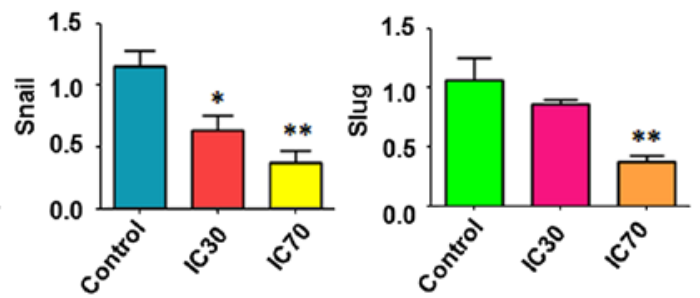

SMMC-7721 GR
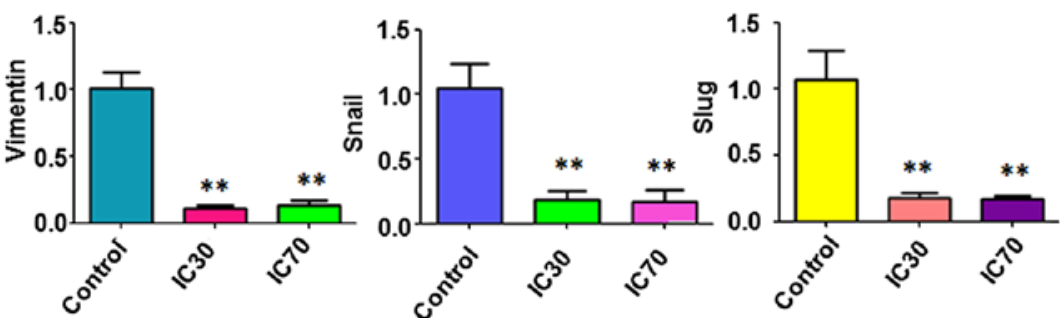

SMMC-7721 GR

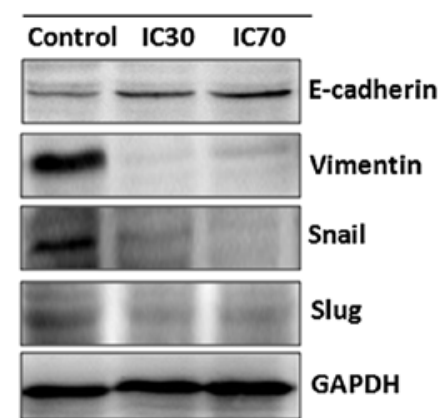

Figure 3. Nutlin-3 reverses EMT to MET in GR HCC cells. (A and B) RT-PCR was conducted to detect the mRNA levels of E-cadherin, Slug, Snail, and vimentin in (A) GR HepG2 and (B) GR SMMC-7721 cells treated with Nutlin-3. ${ }^{*} \mathrm{P}<0.05,{ }^{* *} \mathrm{P}<0.01$ vs. control. (C) Western blot analysis was conducted to measure the abundance of E-cadherin, Snail, Slug, and vimentin proteins in the GR HCC cells treated with Nutlin-3.

A
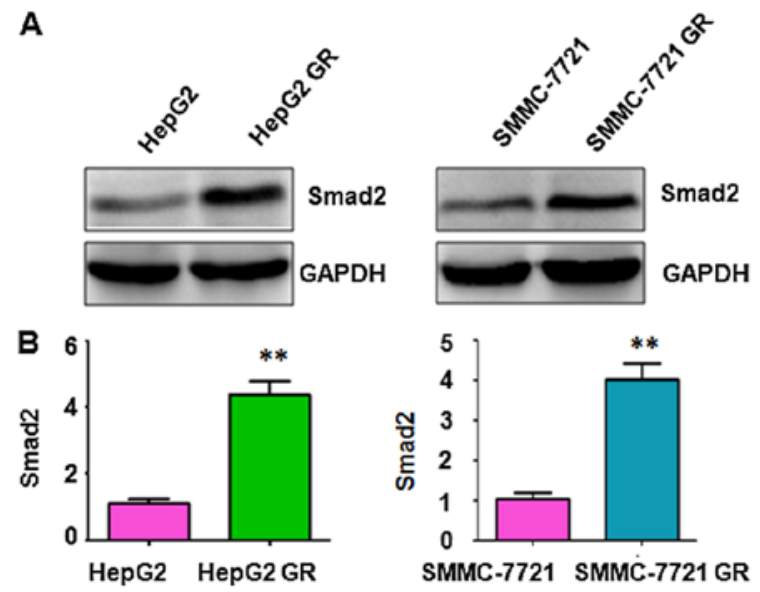

C
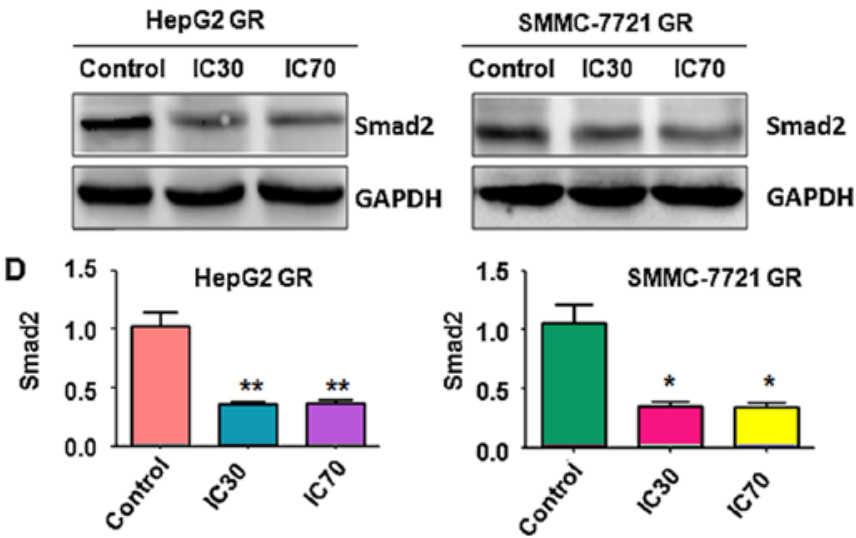

Figure 4. Activation of Smad2 is observed in GR HCC cells. (A) Western blot analysis was conducted to detect the expression of Smad2 in GR HCC cells. (B) Quantitation of results from panel A. ${ }^{* *} \mathrm{P}<0.01$ vs. control. (C) Western blot analysis was conducted to detect Smad2 expression in GR HCC cells treated with Nutlin-3. (D) Quantitative results are illustrated for panel C. ${ }^{*} \mathrm{P}<0.05,{ }^{* *} \mathrm{P}<0.01$ vs. control.

phenotype, suggesting that Nutlin-3 could be a potential treatment agent to target GR-triggered EMT in HCC cells.

Activation of Smad2 is observed in GR HCC cells. Since Smad2 was previously reported to be involved in the EMT process (29), we measured the expression of Smad 2 by western blot analysis. We found higher expression of Smad2 in GR HepG2 and GR SMMC-7721 cells compared with their parental control cells (Fig. 4A and B), suggesting that Smad2 might be involved in GR-induced EMT in HCC cells. It has been reported that 

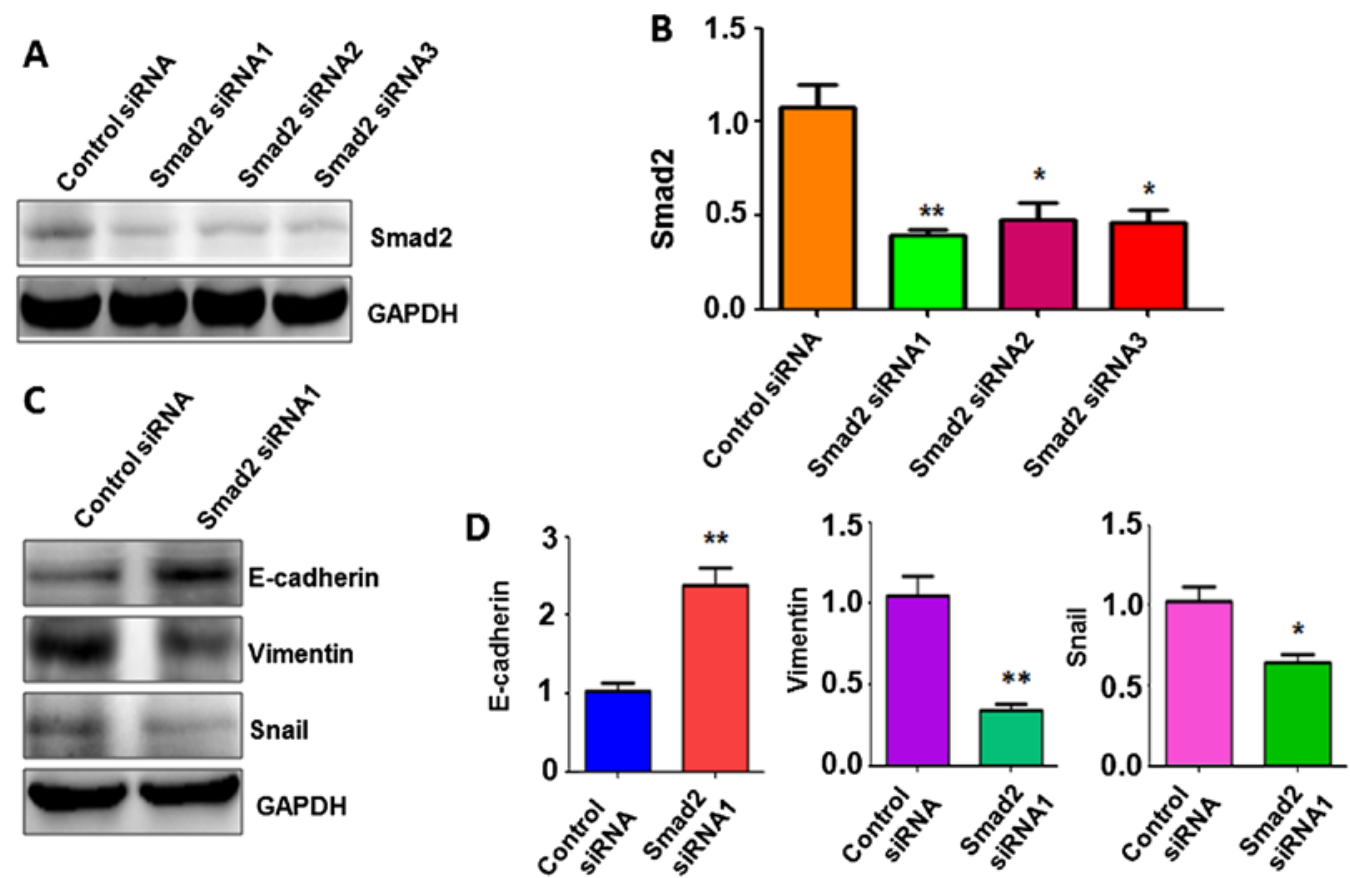

Figure 5. Depletion of Smad2 reverses EMT to MET in GR cells. (A) Western blot analysis of Smad2 in GR HCC cells transfected with different Smad2 siRNAs. (B) Quantitation of results from panel A. ${ }^{*} \mathrm{P}<0.05,{ }^{* *} \mathrm{P}<0.01$ vs. control. (C) Western blot analysis of the expression of E-cadherin, vimentin, and Snail in GR HCC cells treated with Smad2 siRNA1. (D) Quantitative results are illustrated for panel C. ${ }^{*} \mathrm{P}<0.05,{ }^{* *} \mathrm{P}<0.01$ vs. control.

Nutlin-3 prohibited EMT through blocking the phosphorylation of Smad2 in human cancer cells (30). Therefore, we determined whether Nutlin-3 inhibits GR-mediated EMT in HCC cells via targeting Smad2. Consistent with the previous report (29), our western blot analysis revealed that Nutlin-3 inhibited the expression of Smad 2 in both the GR HepG2 and GR SMMC-7721 cells (Fig. 4C and D), indicating that Nutlin-3 suppressed GR-induced EMT partly through inhibition of Smad2 in HCC cells.

Depletion of Smad2 reverses EMT to MET in GR cells. To determine whether Smad2 plays a key role in GR-mediated EMT, we depleted Smad2 using specific siRNAs in the GR HepG2 cells. Western blot analysis was used to detect the efficacy of multiple Smad2 siRNAs on the downregulation of Smad2 in the GR HCC cells. We found that all three Smad2 siRNAs significantly depleted Smad2 expression (Fig. 5A and B). Smad2 siRNA1 was then used for our subsequent studies. To confirm whether depletion of Smad2 regulates GR-induced EMT, we measured the expression of EMT markers by western blot analysis in the GR HCC cells transfected with Smad2 siRNA1. Our results demonstrated that depletion of Smad2 upregulated E-cadherin protein levels, but downregulated the protein levels of vimentin and Snail in the GR HepG2 cells (Fig. 5C and D). These findings suggest that depletion of Smad2 resulted in the reversal of EMT to MET in GR HCC cells.

Depletion of Smad2 inhibits migration and invasion in GR cells. To further characterize the reversal of EMT by depletion of Smad2, we measured cell motility capacities by wound healing, migration and invasion assays. Our wound healing assay results showed that $\mathrm{Smad} 2$ depletion led to a decrease
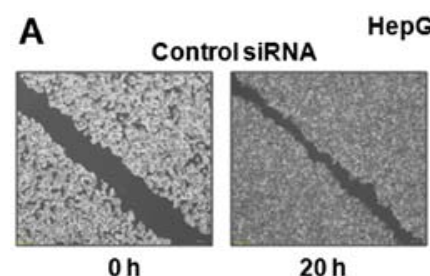

$20 \mathrm{~h}$

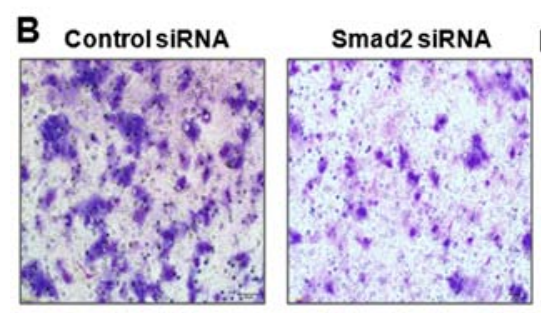

HepG2 GR

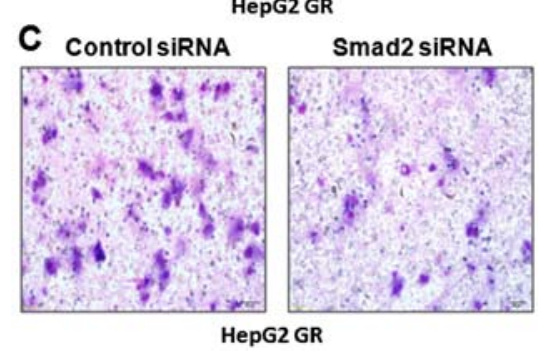

Figure 6. Depletion of Smad2 inhibits the migration and invasion of GR cells. (A) Wound healing assay of GR HepG2 cells transfected with Smad2 siRNA. (B) Migration assay of GR HepG2 cells after Smad2 siRNA transfection. (C) Invasion assay in GR HepG2 cells after Smad2 siRNA treatment. (D) Cell attachment and detachment assays in GR HepG2 cells treated with Smad 2 siRNA. ${ }^{*} \mathrm{P}<0.05$ vs. control.

in the number of cells migrating across the wound (Fig. 6A). In further support of this finding, depletion of Smad2 suppressed cell migration and invasion in the GR HCC 
cells (Fig. 6B and C). In addition, we also performed the cell detachment and attachment assays following Smad2 depletion. We observed that GR HepG 2 cells depleted of Smad 2 displayed reduced detachment and attachment capacity (Fig. 6D). Taken together, our data demonstrated that downregulation of Smad2 retarded cell motility capacities in GR HCC cells, and provides a potential mechanism by which Nutlin-3 suppresses tumor cell growth and metastasis.

\section{Discussion}

In this study, we identified that Nutlin-3 reversed EMT to MET in GR HCC cells in part through downregulation of Smad2. Previous results demonstrated that Nutlin-3 selectively inhibited cell proliferation and enhanced apoptosis by activating the p53 pathway in human cancers including HCC (24). For example, it has been reported that Nutlin-3 enhanced the growth inhibition by doxorubicin and potentiated the apoptotic effects of doxorubicin in HepG2 cells via the disruption of p53-MDM2 binding (31). Moreover, Nutlin-3 was found to inhibit cell proliferation and induce $\mathrm{G}_{0} / \mathrm{G}_{1}$ phase-arrest through downregulation of cyclin D1, cyclin E, CDK2, CDK4, PCNA and E2F-1 and upregulation of p21 and p27 in human HCC cells (32). Furthermore, Shi et al discovered that Nutlin-3 induced apoptosis through downregulation of phospho-Ser392-p53 in HCC cells (33). Recently, they further identified that Nutlin-3 significantly increased the expression levels of interferon- $\gamma$-inducible protein 16 (IFI16) and partially redistributed IFI16 protein to the cytoplasm in SMMC-7721 cells (wild-type p53), but not in Huh-7 (p53 mutant) and Hep3B (p53-null) cells, suggesting that Nutlin-3 controlled the subcellular localization of IFI16 in a p53-dependent manner (34). Nutlin-3 was further reported to potentiate the antiproliferative activity of gefitinib and lapatinib in cancer cells (30). In line with these findings, we found that Nutlin-3 inhibited cell proliferation in GR HepG2 and GR SMMC-7721 cells.

Emerging evidence has revealed that Nutlin-3 could be a useful tool to overcome chemoresistance in human cancer. For example, it has been demonstrated that Nutlin-3 could reverse P-glycoprotein-mediated multidrug resistance in neuroblastoma and rhabdomyosarcoma cell lines (35). Moreover, one study showed that Nutlin-3 sensitized neuroblastoma cells to doxorubicin through upregulation of E2F1 and p73 (36). Similarly, Nutlin-3 activated the p53 pathway and inhibited tumor growth in chemoresistant neuroblastoma cells (37). Notably, Nutlin-3 was considered as a potent enhancer of DR5-selective TRAIL variant D269H/E195R-induced apoptosis in cancer cells (38). Additionally, cisplatin further enhanced Nutlin-3 and DR5-mediated apoptosis (38). Nutlin-3 was also identified to overcome arsenic trioxide resistance and inhibit tumor metastasis via activation of p73 and enhancing mutant p53 degradation mediated by arsenic trioxide in HCC cells (26). These reports indicate that Nutlin-3 is involved in regulating drug resistance of cancer cells. A number of studies havev validated that EMT is associated with drug resistance (10). Indeed, in this study, we observed that GR HCC cells acquired EMT features. Importantly, Nutlin-3 reversed EMT, leading to inhibition of migration and invasion in GR HCC cells. Mechanistically, Nutlin-3 treatment increased
E-cadherin expression and downregulated the expression of vimentin, Snail, and Slug in GR HCC cells.

It has been previously demonstrated that TGF- $\beta$ triggers EMT via governing Smad family expression. One study showed that Nutlin-3 could abolish the decreased in E-cadherin levels induced by TGF- $\beta 1$ in cancer cells (30). Furthermore, this same group found that Nutlin-3 suppressed EMT by inhibition of phosphorylation of Smad2/3, leading to decreased motility of cancer cells (30). Since Smad2 has previously been shown to play an oncogenic role in HCC cells, inhibition of Smad2 could be a potential strategy for treating HCC $(39,40)$. Consistent with this notion, we also identified that Nutlin-3 inhibited the expression of Smad2 in GR HCC cells. Strikingly, depletion of Smad2 inhibited cell migration and invasion in GR cells and regulated EMT marker expression, indicating that Nutlin-3 may reverse GR-mediated EMT partly via downregulation of the Smad2 pathway.

Nutlin-3 exerts its antitumor activity in a p53-dependent manner. In tumors carrying a wild-type p53, Nutlin-3 prevented p53 protein degradation leading to maintained activation of p53, and subsequent induction of p21 and Bax expression, which are regulated by $\mathrm{p} 53$. However, multiple studies also found that Nutlin-3 inhibited cell growth and enhanced apoptosis in a p53-independent manner. For instance, Zheng et al reported that Nutlin-3 disrupted the p73-MDM2 interaction in Huh-7 (p53 mutant) and Hep3B (p53-null) HCC cells and subsequently activated the apoptotic pathway, leading to the increased chemosensitivity to doxorubin (31). This group further found that Nutlin-3 suppressed cell growth and induced $\mathrm{G}_{0} / \mathrm{G}_{1}$ cell cycle arrest in p53-mutant and p53-null HCC cells (32). Similarly, Nutlin-3 was reported to induce apoptosis in Huh-7 cells (33). These reports argued that Nutlin-3 could have anticancer effects against human cancer cells regardless of p53 status. Collectively, Nutlin-3 could be an attractive agent for the treatment of HCC and offers new prospects to overcome chemoresistance. Without a doubt, further in-depth investigation is required to explore the molecular mechanism of Nutlin-3-mediated tumor growth inhibition.

\section{Acknowledgenents}

This study was supported by funding from the Natural Science Program of the Education Office of Anhui Province (KJ2013B147), the Natural Science Research Key Project of Education Office of Anhui Province (KJ2014A152), and the Natural Science Foundation of Zhejiang Province (LY14H160045).

\section{References}

1. Torre LA, Bray F, Siegel RL, Ferlay J, Lortet-Tieulent J and Jemal A: Global cancer statistics, 2012. CA Cancer J Clin 65: 87-108, 2015

2. Siegel RL, Miller KD and Jemal A: Cancer statistics, 2015. CA Cancer J Clin 65: 5-29, 2015.

3. Trovato FM, Tognarelli JM, Crossey MM, Catalano D, Taylor-Robinson SD and Trovato GM: Challenges of liver cancer: Future emerging tools in imaging and urinary biomarkers. World J Hepatol 7: 2664-2675, 2015.

4. Llovet JM, Ricci S, Mazzaferro V, Hilgard P, Gane E, Blanc JF, de Oliveira AC, Santoro A, Raoul JL, Forner A, et al; SHARP Investigators Study Group: Sorafenib in advanced hepatocellular carcinoma. N Engl J Med 359: 378-390, 2008. 
5. Cheng AL, Kang YK, Chen Z, Tsao CJ, Qin S, Kim JS, Luo R, Feng J, Ye S, Yang TS, et al: Efficacy and safety of sorafenib in patients in the Asia-Pacific region with advanced hepatocellular carcinoma: A phase III randomised, double-blind, placebo-controlled trial. Lancet Oncol 10: 25-34, 2009.

6. Qin S, Bai Y, Lim HY, Thongprasert S, Chao Y, Fan J, Yang TS, Bhudhisawasdi V, Kang WK, Zhou Y, et al: Randomized, multicenter, open-label study of oxaliplatin plus fluorouracil/leucovorin versus doxorubicin as palliative chemotherapy in patients with advanced hepatocellular carcinoma from Asia. J Clin Oncol 31 3501-3508, 2013.

7. Zaanan A, Williet N, Hebbar M, Dabakuyo TS, Fartoux L, Mansourbakht T, Dubreuil O, Rosmorduc O, Cattan S, Bonnetain F, et al: Gemcitabine plus oxaliplatin in advanced hepatocellular carcinoma: A large multicenter AGEO study. J Hepatol 58: 81-88, 2013

8. Llovet JM, Villanueva A, Lachenmayer A and Finn RS: Advances in targeted therapies for hepatocellular carcinoma in the genomic era. Nat Rev Clin Oncol 12: 408-424, 2015.

9. Wörns MA and Galle PR: HCC therapies-lessons learned. Nat Rev Gastroenterol Hepatol 11: 447-452, 2014.

10. Wang Z, Li Y, Ahmad A, Azmi AS, Kong D, Banerjee S and Sarkar FH: Targeting miRNAs involved in cancer stem cell and EMT regulation: An emerging concept in overcoming drug resistance. Drug Resist Updat 13: 109-118, 2010.

11. Puisieux A, Brabletz T and Caramel J: Oncogenic roles of EMT-inducing transcription factors. Nat Cell Biol 16: 488-494, 2014.

12. De Craene B and Berx G: Regulatory networks defining EMT during cancer initiation and progression. Nat Rev Cancer 13: 97-110, 2013

13. Mitra A, Mishra L and Li S: EMT, CTCs and CSCs in tumor relapse and drug-resistance. Oncotarget 6: 10697-10711, 2015.

14. Wu Q, Wang R, Yang Q, Hou X, Chen S, Hou Y, Chen C, Yang Y, Miele L, Sarkar FH, et al: Chemoresistance to gemcitabine in hepatoma cells induces epithelial-mesenchymal transition and involves activation of PDGF-D pathway. Oncotarget 4: 1999-2009, 2013.

15. Wang R, Cheng L, Xia J, Wang Z, Wu Q and Wang Z: Gemcitabine resistance is associated with epithelial-mesenchymal transition and induction of HIF-1 $\alpha$ in pancreatic cancer cells. Curr Cancer Drug Targets 14: 407-417, 2014.

16. Güngör C, Zander H, Effenberger KE, Vashist YK, Kalinina T, Izbicki JR, Yekebas E and Bockhorn M: Notch signaling activated by replication stress-induced expression of midkine drives epithelial-mesenchymal transition and chemoresistance in pancreatic cancer. Cancer Res 71: 5009-5019, 2011.

17. Wang Z, Li Y, Kong D, Banerjee S, Ahmad A, Azmi AS, Ali S, Abbruzzese JL, Gallick GE and Sarkar FH: Acquisition of epithelial-mesenchymal transition phenotype of gemcitabine-resistant pancreatic cancer cells is linked with activation of the notch signaling pathway. Cancer Res 69: 2400-2407, 2009.

18. Rieber M and Strasberg-Rieber M: p53 inactivation decreases dependence on estrogen/ERK signalling for proliferation but promotes EMT and susceptility to 3-bromopyruvate in ER $\alpha^{+}$ breast cancer MCF-7 cells. Biochem Pharmacol 88: 169-177, 2014.

19. Lin Y, Mallen-St Clair J, Luo J, Sharma S, Dubinett $S$ and St John M: p53 modulates NF- $\kappa$ B mediated epithelial-to-mesenchymal transition in head and neck squamous cell carcinoma. Oral Oncol 51: 921-928, 2015.

20. Alam SK, Yadav VK, Bajaj S,Datta A,Dutta SK,BhattacharyyaM, Bhattacharya S, Debnath S, Roy S, Boardman LA, et al: DNA damage-induced ephrin-B2 reverse signaling promotes chemoresistance and drives EMT in colorectal carcinoma harboring mutant p53. Cell Death Differ 23: 707-722, 2016.

21. Wade M, Li YC and Wahl GM: MDM2, MDMX and p53 in oncogenesis and cancer therapy. Nat Rev Cancer 13: 83-96, 2013.

22. Khoo KH, Verma CS and Lane DP: Drugging the p53 pathway: Understanding the route to clinical efficacy. Nat Rev Drug Discov 13: 217-236, 2014

23. Van Maerken T, Rihani A, Van Goethem A, De Paepe A, Speleman F and Vandesompele J: Pharmacologic activation of wild-type p53 by nutlin therapy in childhood cancer. Cancer Lett 344: 157-165, 2014.
24. Secchiero P, Bosco R, Celeghini C and Zauli G: Recent advances in the therapeutic perspectives of Nutlin-3. Curr Pharm Des 17: 569-577, 2011.

25. Barone G, Tweddle DA, Shohet JM, Chesler L, Moreno L, Pearson AD and Van Maerken T: MDM2-p53 interaction in paediatric solid tumours: Preclinical rationale, biomarkers and resistance. Curr Drug Targets 15: 114-123, 2014.

26. Zheng T, Yin D, Lu Z, Wang J, Li Y, Chen X, Liang Y, Song X, Qi S, Sun B, et al: Nutlin-3 overcomes arsenic trioxide resistance and tumor metastasis mediated by mutant p53 in hepatocellular carcinoma. Mol Cancer 13: 133, 2014.

27. Moran DM and Maki CG: Nutlin-3a induces cytoskeletal rearrangement and inhibits the migration and invasion capacity of p53 wild-type cancer cells. Mol Cancer Ther 9: 895-905, 2010.

28. Wang R, Li Y, Hou Y, Yang Q, Chen S, Wang X, Wang Z, Yang Y, Chen C, Wang Z, et al: The PDGF-D/miR-106a/Twist1 pathway orchestrates epithelial-mesenchymal transition in gemcitabine resistance hepatoma cells. Oncotarget 6: 7000-7010, 2015.

29. Saitoh M: Epithelial-mesenchymal transition is regulated at post-transcriptional levels by transforming growth factor- $\beta$ signaling during tumor progression. Cancer Sci 106: 481-488, 2015.

30. Wu Y, Fu Y,Zheng L, Lin G, Ma J, Lou J,Zhu H, He Q and Yang B: Nutlin-3 inhibits epithelial-mesenchymal transition by interfering with canonical transforming growth factor- $\beta 1-$ Smad-Snail/Slug axis. Cancer Lett 342: 82-91, 2014.

31. Zheng T, Wang J, Song X, Meng X, Pan S, Jiang H and Liu L: Nutlin-3 cooperates with doxorubicin to induce apoptosis of human hepatocellular carcinoma cells through p53 or p73 signaling pathways. J Cancer Res Clin Oncol 136: 1597-1604, 2010.

32. Wang J, Zheng T, Chen X, Song X, Meng X, Bhatta N, Pan S, Jiang $\mathrm{H}$ and Liu L: MDM2 antagonist can inhibit tumor growth in hepatocellular carcinoma with different types of p53 in vitro. J Gastroenterol Hepatol 26: 371-377, 2011.

33. Shi X, Liu J, Ren L, Mao N, Tan F, Ding N, Yang J and Li M: Nutlin-3 downregulates p53 phosphorylation on serine392 and induces apoptosis in hepatocellular carcinoma cells. BMB Rep 47: 221-226, 2014.

34. Shi XL, Yang J, Mao N, Wu JH, Ren LF, Yang Y, Yin XL, Wei L, Li MY and Wang BN: Nutlin-3-induced redistribution of chromatin-bound IFI16 in human hepatocellular carcinoma cells in vitro is associated with p53 activation. Acta Pharmacol Sin 36: 252-258, 2015.

35. Michaelis M, Rothweiler F, Klassert D, von Deimling A, Weber K, Fehse B, Kammerer B, Doerr HW and Cinatl J Jr: Reversal of P-glycoprotein-mediated multidrug resistance by the murine double minute 2 antagonist nutlin-3. Cancer Res 69: 416-421, 2009.

36. Peirce SK and Findley HW: The MDM 2 antagonist nutlin- 3 sensitizes p53-null neuroblastoma cells to doxorubicin via E2F1 and TAp73. Int J Oncol 34: 1395-1402, 2009.

37. Van Maerken T, Ferdinande L, Taildeman J, Lambertz I, Yigit N, Vercruysse L, Rihani A, Michaelis M, Cinatl J Jr, Cuvelier CA, et al: Antitumor activity of the selective MDM2 antagonist nutlin-3 against chemoresistant neuroblastoma with wild-type p53. J Natl Cancer Inst 101: 1562-1574, 2009.

38. Meijer A, Kruyt FA, van der Zee AG, Hollema H, Le P, ten Hoor KA, Groothuis GM, Quax WJ, de Vries EG and de Jong S: Nutlin-3 preferentially sensitises wild-type p53-expressing cancer cells to DR5-selective TRAIL over rhTRAIL. Br J Cancer 109: 2685-2695, 2013.

39. Zheng X, Gai X, Han S, Moser CD, Hu C, Shire AM, Floyd RA and Roberts LR: The human sulfatase 2 inhibitor 2,4-disulfonylphenyl-tert-butylnitrone (OKN-007) has an antitumor effect in hepatocellular carcinoma mediated via suppression of TGFB1/SMAD2 and Hedgehog/GLI1 signaling. Genes Chromosomes Cancer 52: 225-236, 2013

40. Wang J, Liu G, Li Q, Wang F, Xie F, Zhai R, Guo Y, Chen T, Zhang N, Ni W, et al: Mucin1 promotes the migration and invasion of hepatocellular carcinoma cells via JNK-mediated phosphorylation of Smad2 at the C-terminal and linker regions. Oncotarget 6: 19264-19278, 2015. 